\title{
Successful Treatment For a Young Patient With Multiple Synchronous Breast And Lung Carcinoma: A Case Report
}

\section{Mengfan Zhao}

The Affiliated Hospital of Southwest Medical University

Lusha Bi

The Affiliated Hospital of Southwest Medical University

Bin Wu ( $\square$ wwbb129@sina.cn )

The Affiliated Hospital of Southwest Medical University

\section{Case report}

Keywords: Breast adenocarcinoma, lung adenocarcinoma, multiple primary malignancies

Posted Date: November 20th, 2020

DOI: https://doi.org/10.21203/rs.3.rs-111312/v1

License: (c) (i) This work is licensed under a Creative Commons Attribution 4.0 International License.

Read Full License 


\section{Abstract}

Introduction Reports of synchronous multiple primary cancers in patients with breast cancer have recently been increasing because of progress in radiographic diagnostic techniques. Multiple primary cancers in patients with breast cancer mainly occur in the lung and endometrium.

Case report We report a 37-year-old female patient diagnosed with synchronous double primary cancers, namely invasive ductal carcinoma of the left breast and invasive carcinoma of inferior lobe of the right lung based on the pathological examination. After MDT, we gave her both lung and breast surgery then adjuvantchemothrapy(AC-T) and EGFR-TKI (Gefitinib). We followed this patient until October, 2020, the patient was healthy and with no clinical or radiologic signs of recurrent or metastatic disease at the time of this writing.

Discuss The pathogenesis of MPCs is still unclear, which is closely related to many factors, such as genetic, immune, iatrogenic, endocrine, lifestyle. The diagnosis depends on chest CT and Pathology examination. And there is no standard treatment guideline for MPCs. The specific therapy plan should be made after MDT discussion.

Conclusion Multiple synchronous breast and lung adenocarcinoma in one patient dose not like metastatic carcinoma. If the treatment is in time, the patient can also get a good prognosis.

\section{Introduction}

Breast cancer is the most common malignant tumors in Chinese woman and seriously endanger their health ${ }^{[1]}$. With the progress in radiographic diagnostic techniques, It is no more rarely seen a patient with multiple primary cancers who suffered from breast cancer synchronously. Multiple primary cancers in patients with breast cancer mainly occur in the lung and endometrium. Many patients were diagnosed breast cancer first because the breast tumors are more superficial than others so the patients can find them by themselves, then they usually find the second primary tumor by preoperation radiographic examination. This paper reports a young patient suffered from multiple synchronous breast and lung adenocarcinoma.

\section{Case Report}

A 37-year-old premenopausal woman was admitted to hospital on March 28, 2019 for finding a mass in her left breast for more than 5 months. There was no past history for her. Physical examination showed a mass of about $2 \star 2 \mathrm{~cm}$ can be palpated in the upper inner quadrant of her left breast (11 o'clock direction), which is hard, irregular in shape, unclear in boundary, and still movable. A $1 * 1 \mathrm{~cm}$ enlarged lymph node can be palpated in her left axilla, and no definite enlarged lymph node can be found in right axilla and the upper and lower clavicles of both sides. Breast ultrasound showed that the size of the left breast mass was about $2.0 * 1.7 * 0.8 \mathrm{~cm}$ in size, and was located at about 10-11 o'clock direction of the left breast, approximately $0.8 \mathrm{~cm}$ away from the skin and $2.5 \mathrm{~cm}$ away from the nipple, Which was diagnosed BI- 
RADS 4C. Multiple hypoechoic could be found in bilateral axillary, and the maximum in left side was about $1.2 * 0.5 \mathrm{~cm}$ (Fig. 1). Mammography showed a irregular high-density mass located in the posterior part of upper quadrant of left breast, with a range of about $2.1 * 1.9 \mathrm{~cm}$, and also was diagnosed BI-RADS 4C(Fig. $2 \mathrm{a}$ and 2b). Breast MRI told that the size of left breast mass was about $2.1 * 1.2 * 2.2 \mathrm{~cm}$, with lobulated shape and spicules of margin, which was diagnosed BI-RADS 4C(Fig. 3). Then we gave her core needle biopsy for left breast mass and fine needle aspiration for left axilla lymph node, the result showed left breast mass was malignancy and the axilla lymph node was negative. Immunohistochemistry: Invasive adenocarcinoma of left breast(who $\mathbb{\text { grade}})$, ER $(+, 80 \%), \mathrm{PR}(+, 70 \%), \mathrm{HER} 2(2+), \mathrm{GATA}-3(+), \mathrm{E}-$ cad (+), p120 (membrane +), calponin (part +), p63 (part +), Ki-67 (+, 30\%), p53 (+, 20\%), FISH examination showed negative. Chest CT: $1.3 \mathrm{~cm}$ GGN was discovered in the anterior basal segment of the lower lobe of right lung, with unclear boundary(Fig. 4). Bone scan: no obvious abnormality was found. Because the uncertainty of this case, we gave her MDT. During MDT discussing, There seemed to be a consensus that the lung nodule was most likely a primary lung carcinoma.

Therefore, the patient was transferred to the department of chest surgery for pulmonary surgery. Videoassisted thoracoscopic wedge-shaped resection of the right lower lobe nodule was performed on April 9, 2019. The results showed that the right lower lobe nodule was consistent with adenocarcinoma. Based on the result, the right lower lobe resection and lymph nodes dissection were performed next. Postoperative pathological examination told tumor size was about $2.5 * 2 \mathrm{~cm}$ and located in the lower lobe of the right lung. Lymph node examination showed: peribronchial lymph nodes $(0 / 1)$, group 2-4 lymph nodes(0 / 2), group 7 lymph nodes(1 / 8), group 8 lymph nodes(0 / 1), group 11 lymph nodes(0 / $5)$, group 12 lymph nodes $(0 / 2)$. the cutting edge was free, no nerve invasion, no pulmonary membrane involvement, no airway spread. Immunohistochemistry: CK (diffuse +), CK5 / 6 (-), P40 (-), CK7 (diffuse +), CK20 (-), napsina (+), TTF-1 (diffuse +), Ki-67 (+, 10\%), p53 (+, 80\%)(Fig. 5). Genetic examination showed EGFR: 21 L858R mutation, supported the diagnosis of primary adenocarcinoma of right lung. After pulmonary surgery, she was transferred to our department and then we gave her mastectomy and sentinel lymph node biopsy ( 0 / 5) for left breast cancer on May 5, 2019.Postoperative pathological examination told: left breast invasive adenocarcinoma (WHO grade区), tumor size is about 2.5 * 2 * $1.5 \mathrm{~cm}$; ductal carcinoma in situ: about $40 \%$; specimen margin: all negative; lymphatic vascular invasion: found; nerve invasion: not found. Immunohistochemistry: ER (+, 90\%), PR (+, 90\%), HER2 (2 +), E-cad (+), p120 (membrane +), AR (-), CK5 / 6 (myoepithelial loss), EGFR (-), GATA-3 (diffuse +), Ki-67 (+, 20\%), p53 $(+, 5 \%)$, SMA (myoepithelial loss), FISH examination showed negative(Fig. 6).

After operation, we completed 8 cycles of adjuvant chemotherapy of epirubicin $90 \mathrm{mg} / \mathrm{m}^{2}$ (total $130 \mathrm{mg}$ ), IVP / IV, GTT, D1; cyclophosphamide $600 \mathrm{mg} / \mathrm{m}^{2}$ (total $900 \mathrm{mg}$ ), IVP / IV, GTT, D1; followed docetaxel $90 \mathrm{mg} / \mathrm{m}^{2}$ (total $130 \mathrm{mg}$ ), IVP / IV, GTT, D1 three week of a cycle, and gefitinib was given to lung adenocarcinoma EGFR targeted therapy, and tamoxifen and goserellin were continued after chemotherapy for endocrine therapy. Follow-up to September 2020, no tumor recurrence and metastasis.

\section{Discuss}


Multiple primary cancers (MPCs) are defined as two or more kinds of primary malignant tumors occurring synchronously or heterochronously in the same patient. The diagnostic criteria are as follows: 1) Each tumor must be confirmed as malignant by histopathology; 2) Each tumor must be separated and independent in its location; 3) Suspected metastatic or recurrent cancer must be excluded. According to the interval of occurrence time, MPCs can be divided into synchronous type (the interval time between the first primary cancer is less than 6 months) and heterochronous type (the interval time is more than 6 months) ${ }^{[2-4]}$. According to the above diagnostic criteria, this case belongs to synchronous type. The incidence rate of MPCs is low, about $0.73 \%-11.70 \%$, the ratio of male to female is $0.986: 1^{[5-6]}$. The pathogenesis of MPCs is still unclear, which is closely related to many factors, such as genetic factors, immune factors, iatrogenic factors, endocrine factors, lifestyle. In recent years, the incidence rate of multiple cancers had been increasing, this may be related to the progress of radiographic diagnostic techniques, and also environmental factors and the improvement of patients' healthy consciousness.

Breast cancer and lung cancer are two common malignant tumors occurring in women and there is a certain correlation between them. The incidence rate of lung cancer in breast cancer patients is 2 times higher than that in healthy people $\mathrm{e}^{[7]}$. Some studies have shown that estrogen is also closely related to the occurrence of lung cancer which has been proved to be associated with breast cancer, and many lung cancer cells are accompanied with ER over-expression ${ }^{[8]}$. Lung is also one of the most frequent metastatic sites of breast cancer.

After the diagnosis of pulmonary malignancies in breast cancer patients, the key of making next treatment is defining the source of the pulmonary malignancies. Chest CT is the most used method of defining the source of the pulmonary tumors. The primary malignant pulmonary tumors generally present in CT images as solitary nodules with irregular edges, which can present lobulation, spicule sign, pleural indentation, while metastatic pulmonary nodules often show multiple nodules in both sides lungs with smooth edges. The gold standard of source defining depends on the results of pathological examination. Recently, It had been reported that the expression of GATA-3 in pathology was mainly used to support that the tumors were derived from mammary gland or urinary epithelium ${ }^{[9-10]}$. In other words, GATA-3 was highly expressed in breast cancer so it was also highly expressed in tumors metastasized by breast cancer. In addition, TTF-1 and NapsinA both have high diagnostic value in primary lung adenocarcinoma, but they are almost not expressed in breast cancer. Therefore, immunohistochemistry can be used to further determine whether the tumor is primary or metastatic ${ }^{[11]}$.

The treatment principle of MPCs is the same as that of primary cancer, so radical surgical resection is the first choice. If the patients can tolerate surgery, and there is no certain systemic metastasis, surgical treatment should be considered first, and then chemotherapy, radiotherapy, targeted therapy should be carried out according to the actual stage of tumors ${ }^{[12-13]}$. Mastectomy is the best choice for those patients instead of Breast conservation surgery. At present, there is no standard guideline for MPCs of breast and lung adenocarcinoma patients. The specific therapy plan should be made after MDT discussion by experienced experts from the department of breast surgery, chest surgery and oncology. 


\section{Conclusion}

Although the diagnosis rate of MPCs is constantly improving recently, there are still many primary hospitals misdiagnose the patients and delay their optimal therapy opportunity due to the lack of examination methods and diagnostic experience, resulting in the occurrence of metastatic cancer, eventually influencing the prognosis of patients. The treatment and prognosis of MPCs patients are completely different from those of metastatic cancers patients. Therefore, surgeons should enhance the awareness of diagnosis of MPCs, especially for the elderly patients with family history of tumor diseases, chemotherapy or radiotherapy history, low immune function, and poor physical health. Reasonable examination methods should be used to ensure that every patient with MPCs will not be left, and MDT discussion is supposed to be carried out to make individualized treatment plan for specific patients, so that patients with MPCs can get the best prognosis.

\section{Abbreviations List}

MPCs: Multiple primary cancers

AC-T: Doxorubicin+ Cyclophosphamide- Docetaxel

EGFR-TKI: Epidermal growth factor receptor-tyrosine kinase inhibitor

MDT: Multiple disciplinary team

ER: Estrogen receptor

GGN: Ground glass nodules

\section{Declarations}

\section{Author's contributor}

Zhao Mengfan conceived, designed and wrote the manuscript. Bi Lusha collected the patient's information. Wu Bin carried out manuscript revision.All authors approved the final version of the manuscript.

\section{Acknowledgement}

We thank Dr.Huaiquan Zuo for enlightening advices.

\section{Funding}

This paper had no funding supported.

\section{Availability of data and materials}


The raw data are available upon request to the corresponding author and/or to the first author.

\section{Ethics approval and consent to participate}

This study was approved by the ethical committee review board of South West Medical University.

\section{Consent for publication}

The patient has signed informed consent of this paper and agreed to publish her case.

\section{Competing interests}

The authors declare that they have no competing interests.

\section{References}

1. Chen W, Zheng R, Baade PD, Zhang S, Zeng H, Bray F, Jemal A, Yu XQ , He J.Cancer statistics in China, 2015. CA Cancer J Clin. 2016;66(2):115-32.doi: 10.3322/caac.21338. Epub 2016 Jan 25.

2. Warren $S$, Gates O.Multiple primary malignant tumors:a survey of the literature and a statistical study[J].Am J Cancer, 1932, 16:1358-1414.

3. Santangelom L, Criscitiello C, Renda A,et al.Immunosupression and multiple primary malignancies in kidney-transplanted patients: a Single-Institute study[J]. Biomed Res Int囚2015ヌ2015:183523.

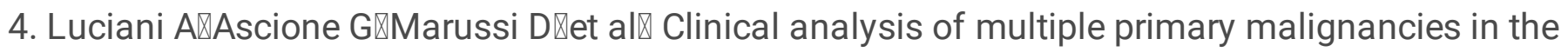

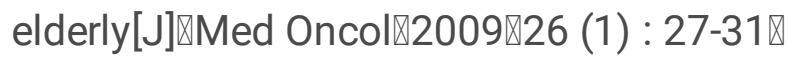

5. IZMMLOWICZB,KORNAFEL J, BLASZCZYK J.Multiple neoplasms among cervical cancer patients in the material of the lower silesian can cerregistry[J].Adv Clin Exp Med, 2014, 23 (3) :433-440.

6. Shang CY, Zhao YN. Analysis of clinical characteristics and prognostic risk factors of multiple cancers [J]. Guangxi Medical College, 2017,09:1299-1303.

7. MELLEMKJAER LهFRIISS $\triangle O L S E N J H \rrbracket e t$ al『Risk of second cancer among women with breast cancer[J]『Int J Cancer囚2006ه118 ( 9 ) : 2285-2292》

8. Mah V囚Marquez D\Alavi M『et al. Expression levels of estrogen receptor beta in conjunction with

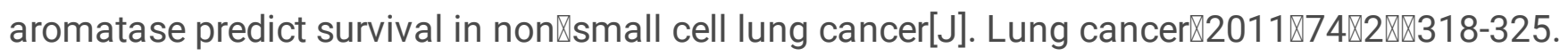

9. Cimino Mathews A囚Subhawong AP『Illei PB囚et al. GATA3 expression in breast carcinoma\utility in

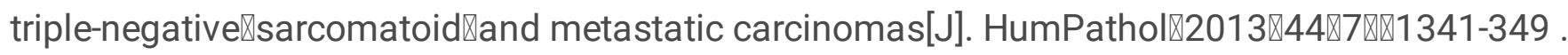

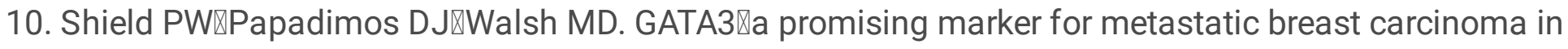
serous effusion specimens[J]. Cancer Cytopathol $₫ 2014 \rrbracket 122 \rrbracket 4 \rrbracket \bigotimes 307-312$.

11. Zhang Wenhua, Xiang Jingjing, sun Lijuan, et al. Comparison of pathological diagnostic value of TTF-1 and napsina in lung adenocarcinoma [J]. Journal of clinical and Experimental Pathology, 2016, 32 (9): 1028-1032. 
12. Li Wei, Zhan Youqing, Li Guohui. Clinical analysis of 156 cases of double primary carcinoma [J]. Chinese Journal of oncology, 1996, 18: 296-298

13. Pang Lijun, Cao Cheng, Hua Yijia, Zeng Tianyu, Yang Fan, sun Chunxiao, Huang Xiang, Yin Yongmei, Li Wei. A case report of breast cancer and lung cancer [J]. Journal of Nanjing Medical University (NATURAL SCIENCE EDITION), 2019,11:1696-1698

\section{Figures}

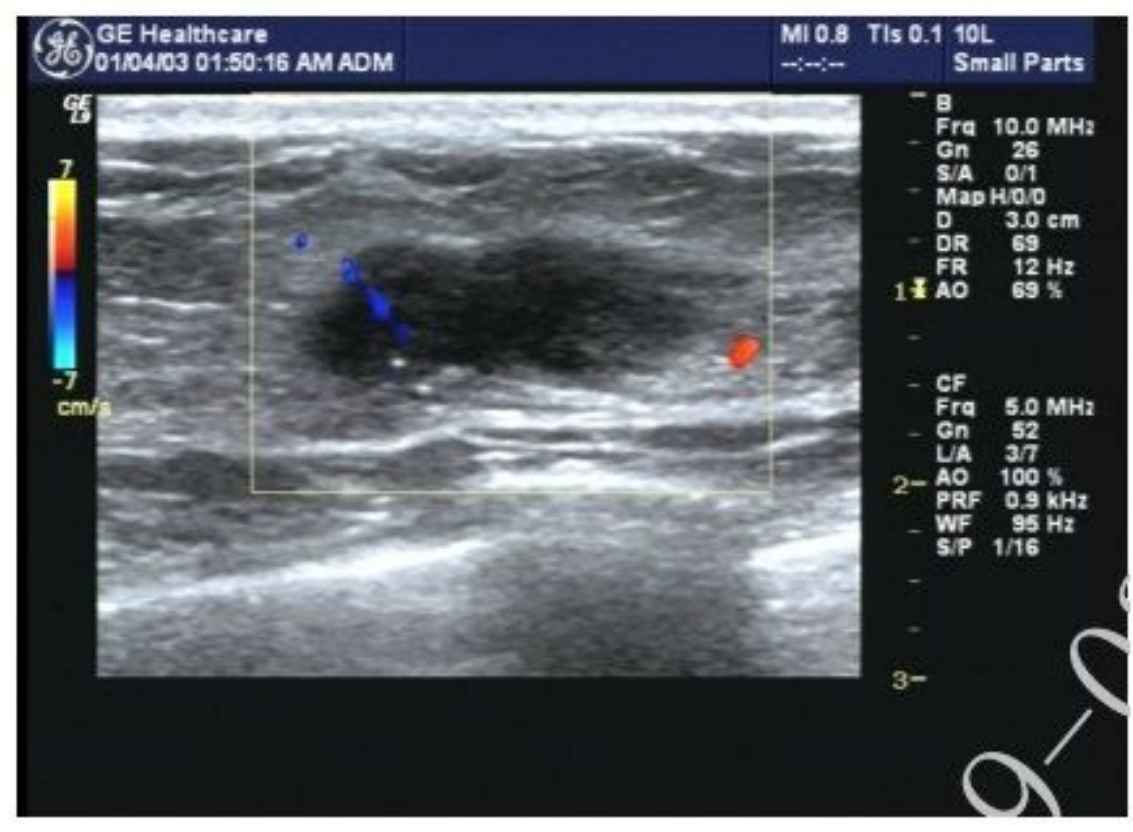

\section{Figure 1}

\section{Breast Ultrasound}

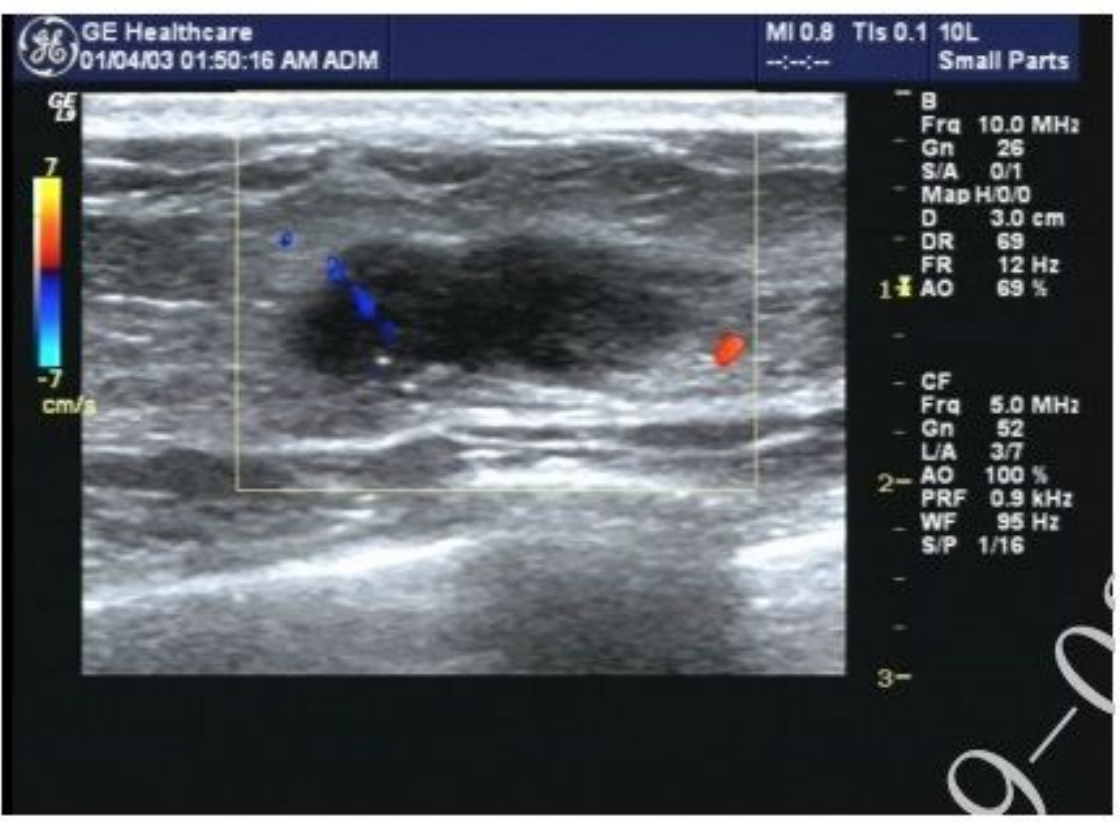


Figure 1

Breast Ultrasound

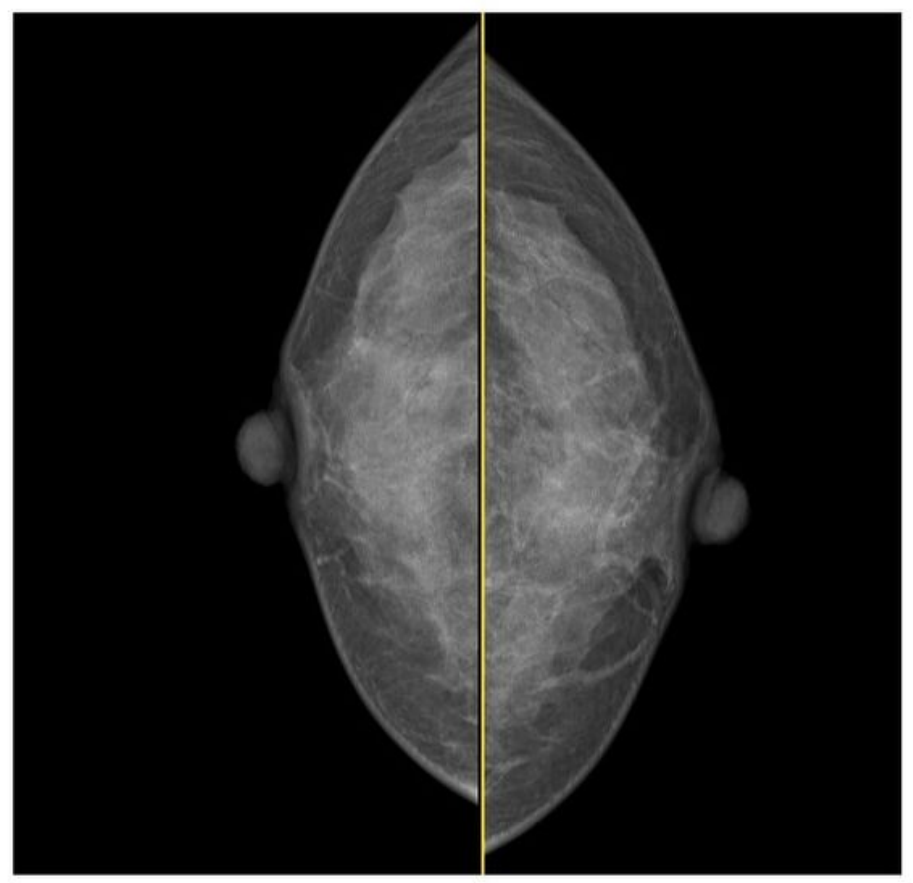

$2 a$

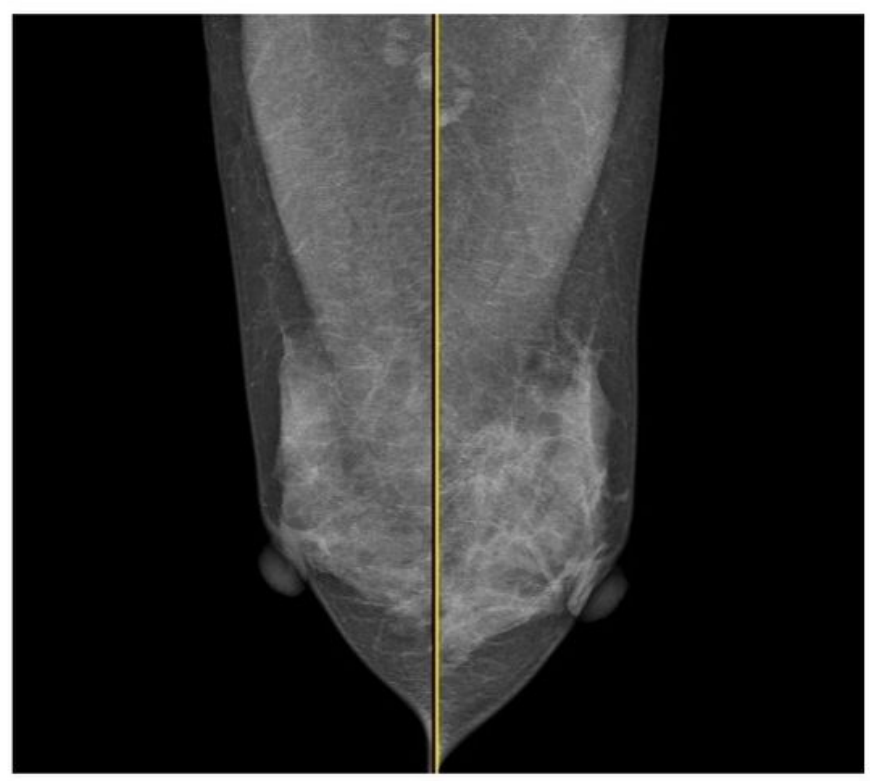

$2 b$

Figure 2

2a Breast mammography (cc) 2b Breast mammography (mlo)
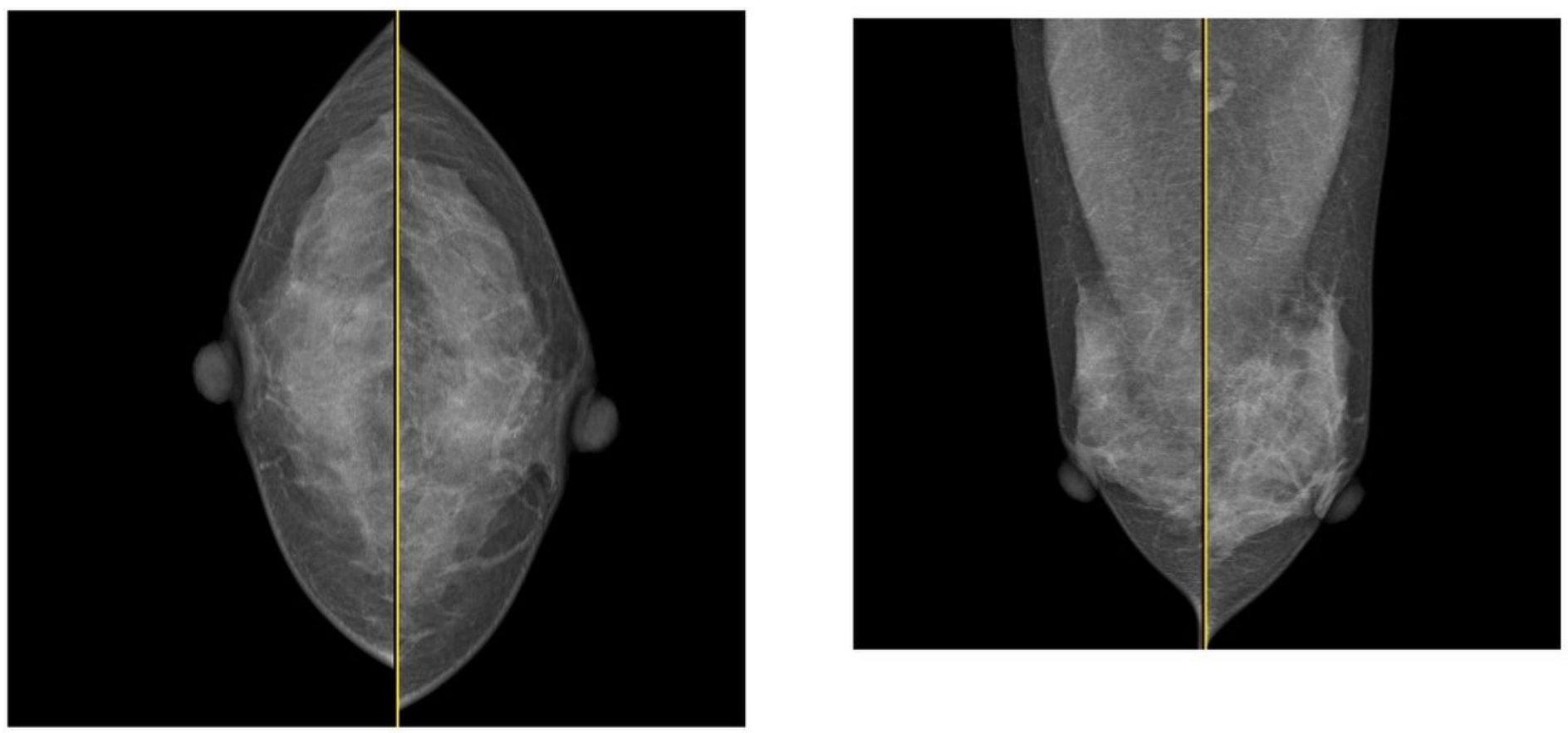

$2 a$

$2 b$

Figure 2 
2a Breast mammography (cc) 2b Breast mammography (mlo)

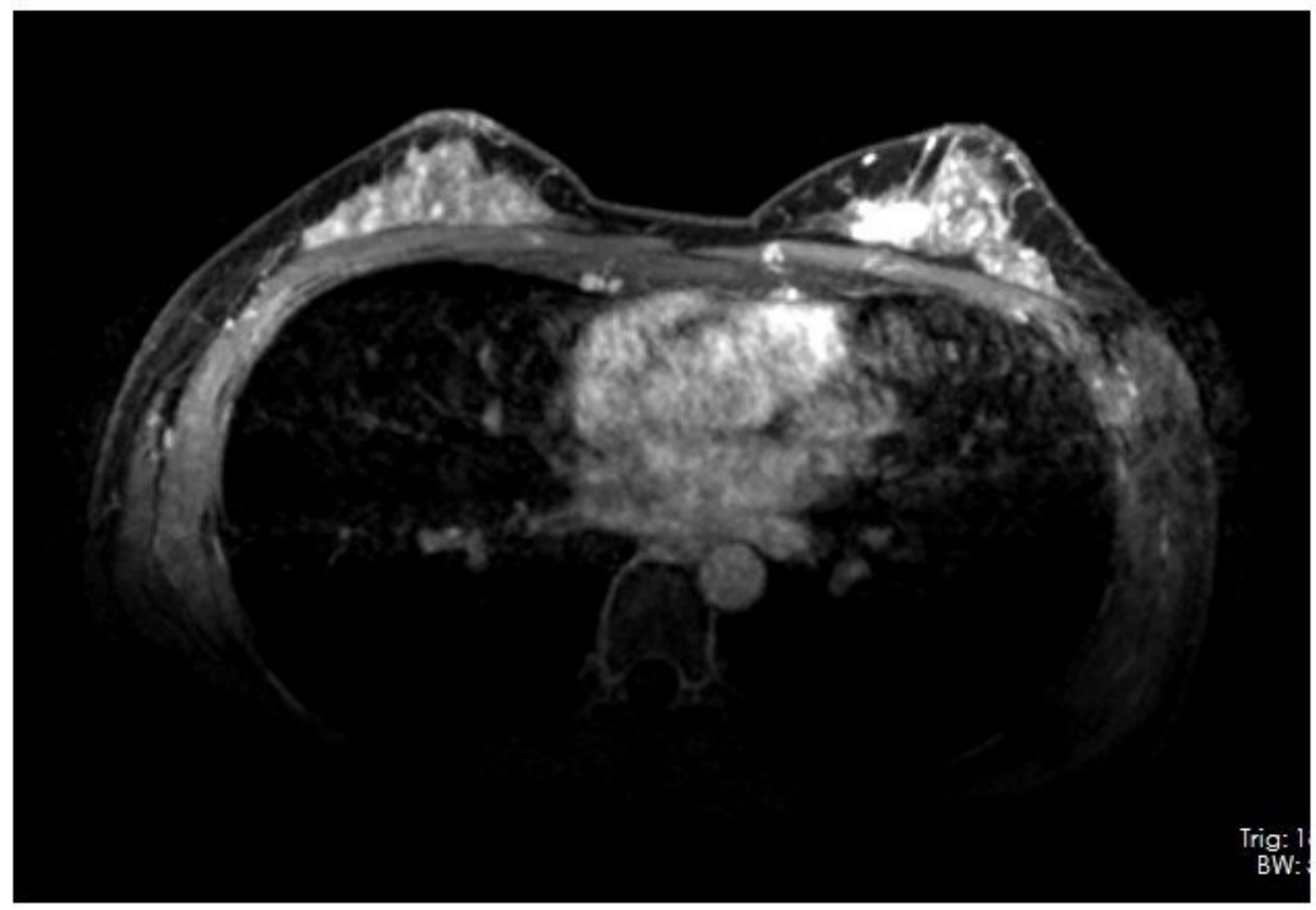

\section{Figure 3}

Breast MRI 


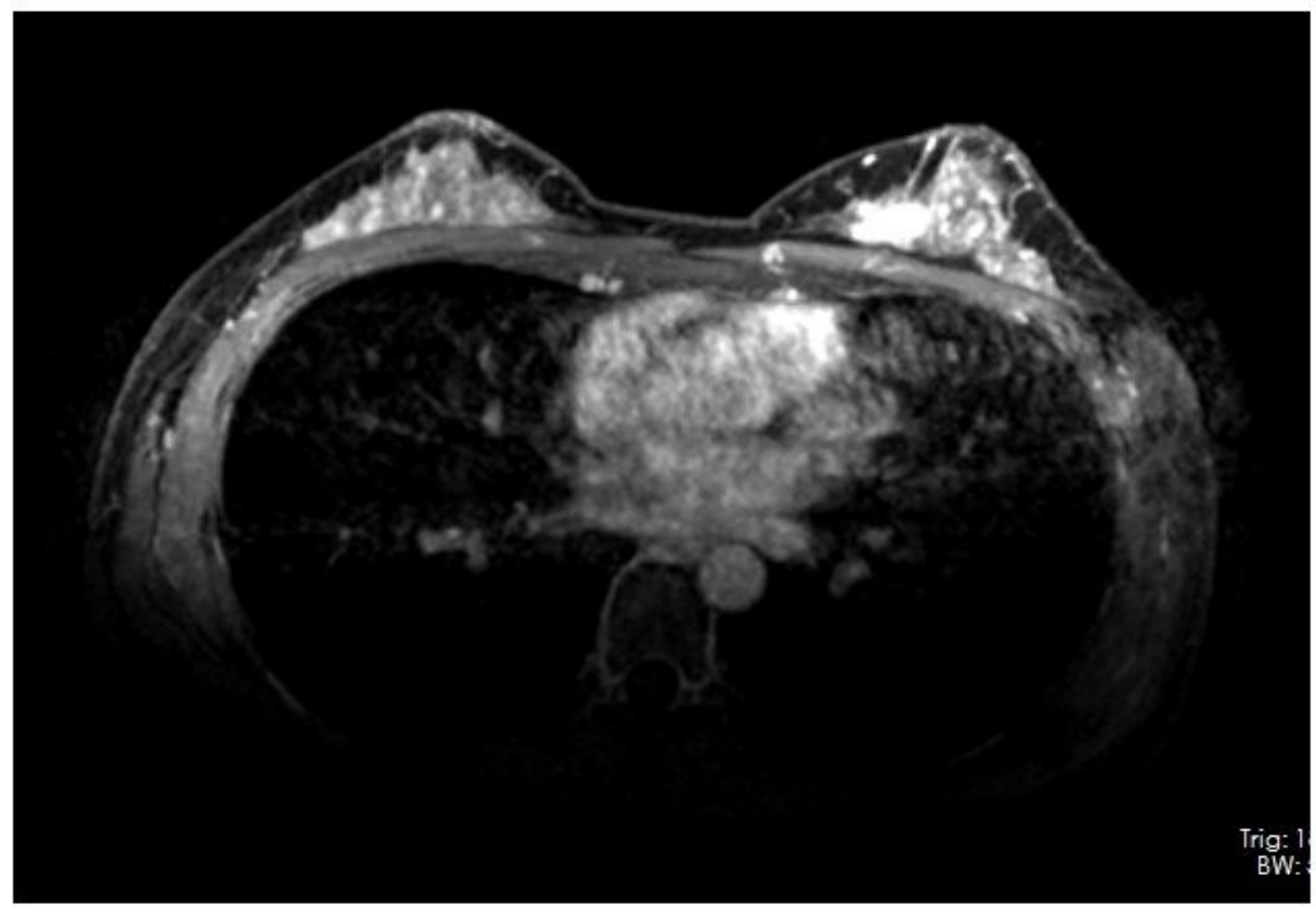

Figure 3

Breast MRI 


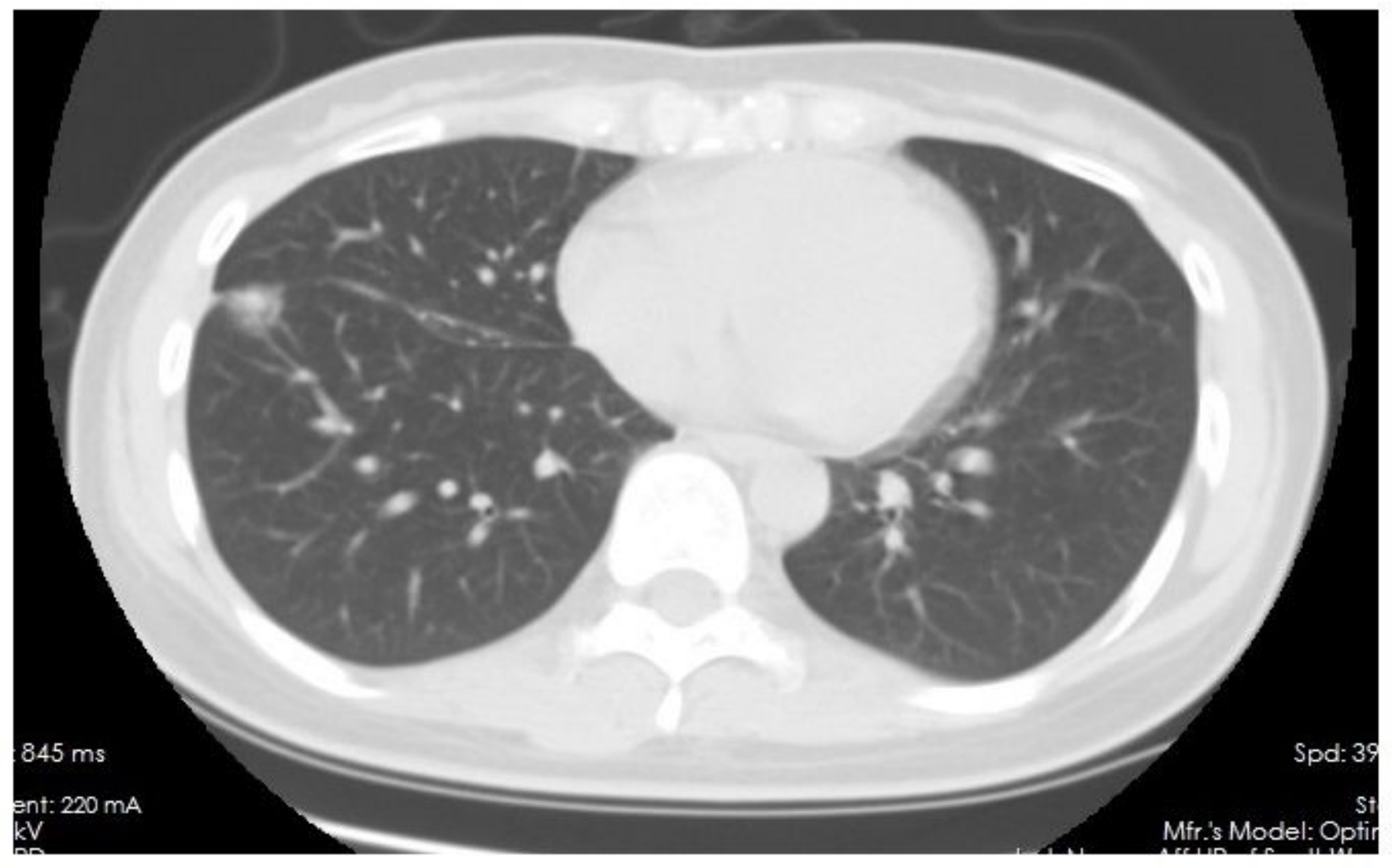

Figure 4

Chest CT 


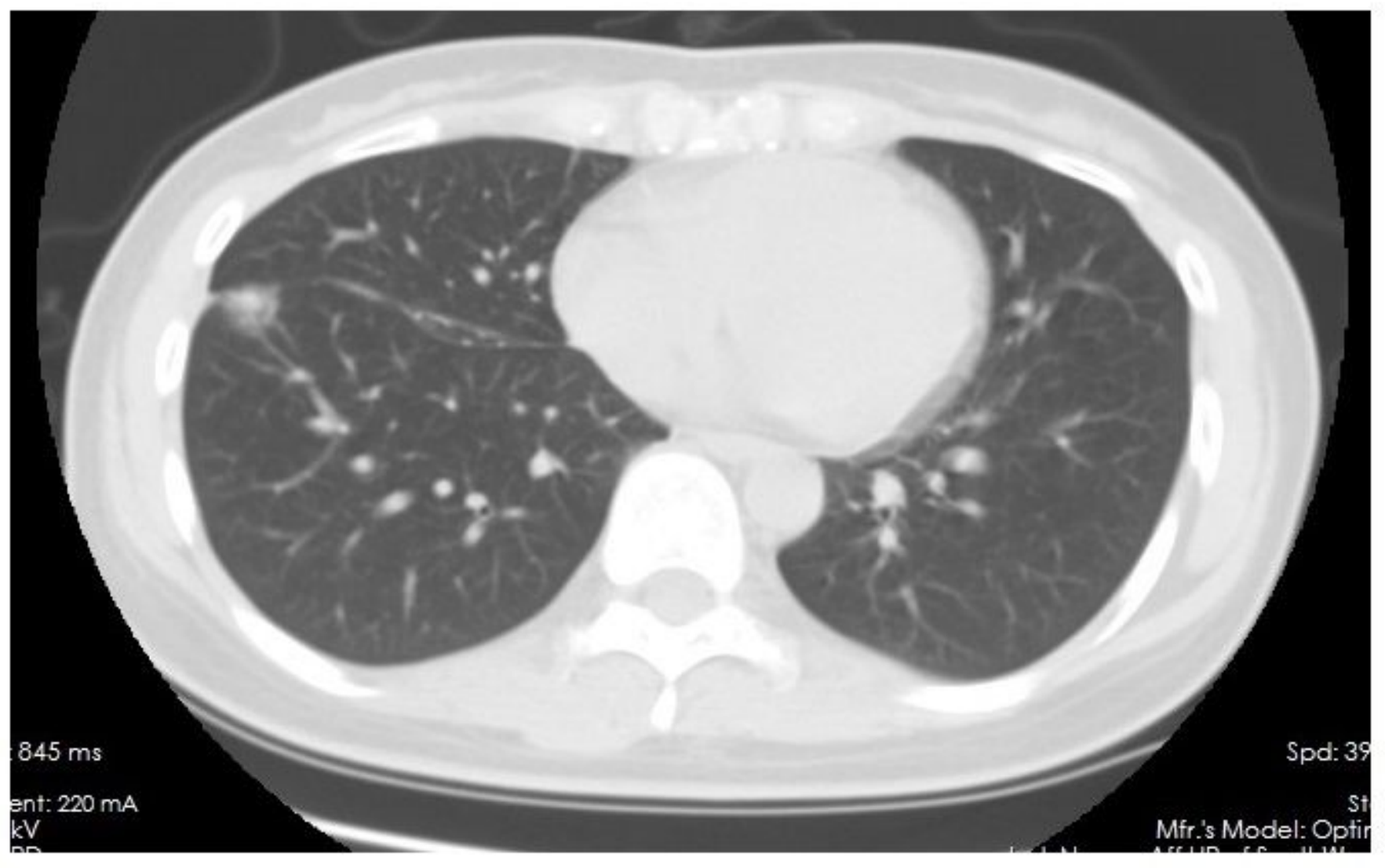

Figure 4

Chest CT

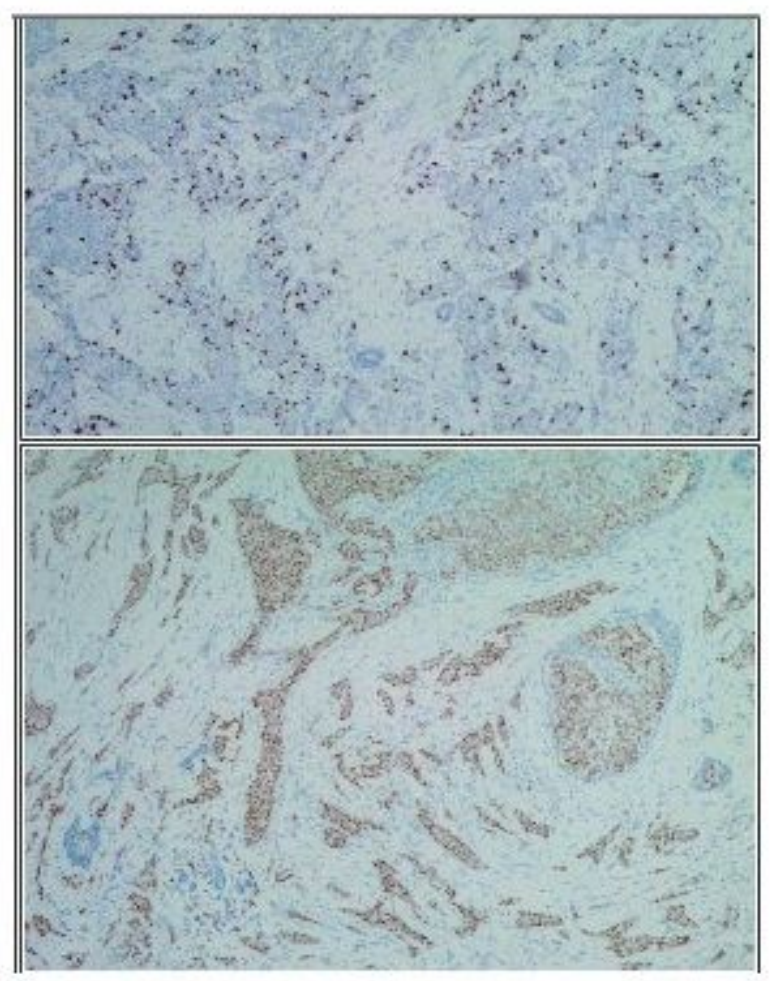

Figure 5 
Immunohistochemistry of breast tumor

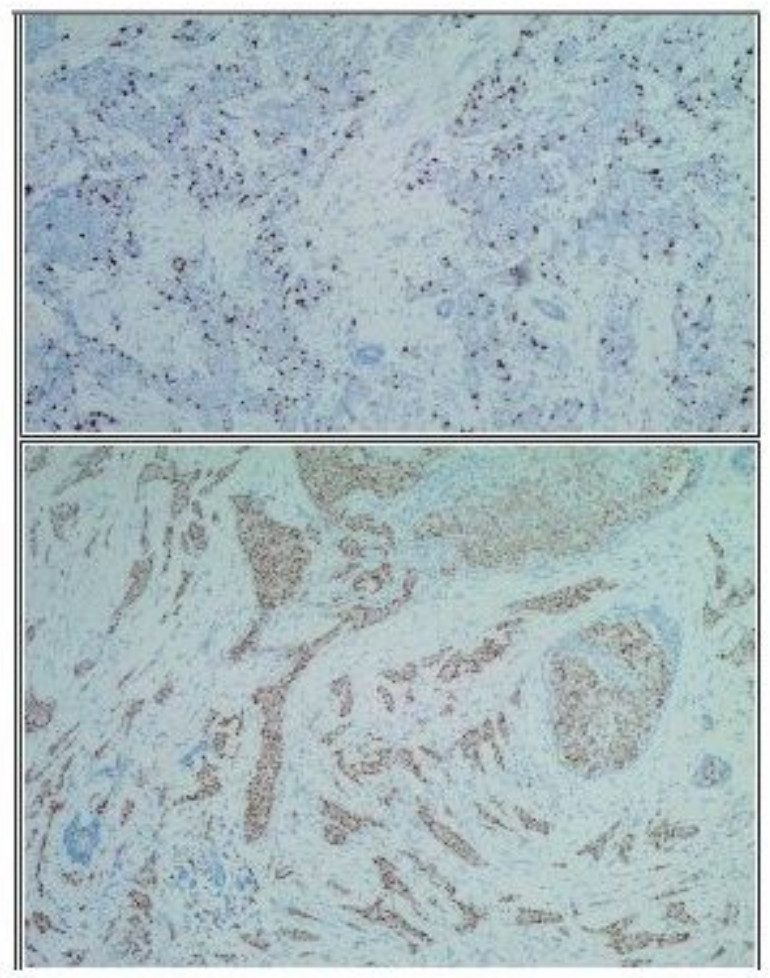

Figure 5

Immunohistochemistry of breast tumor

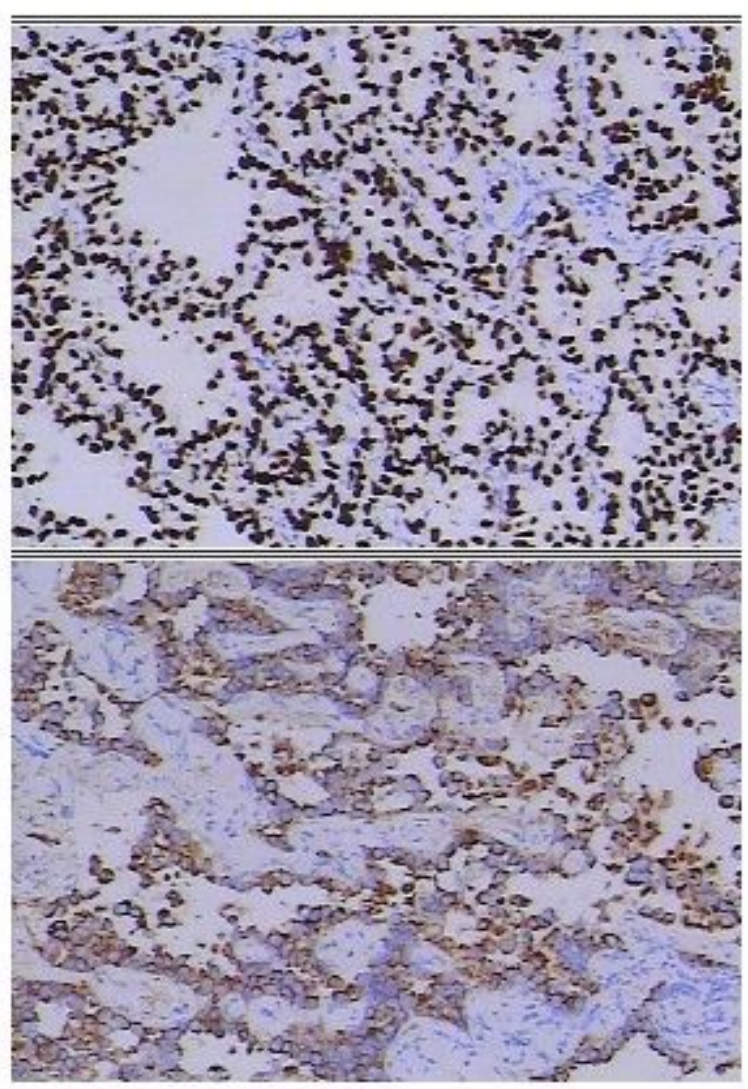


Figure 6

Immunohistochemistry of lung tumor

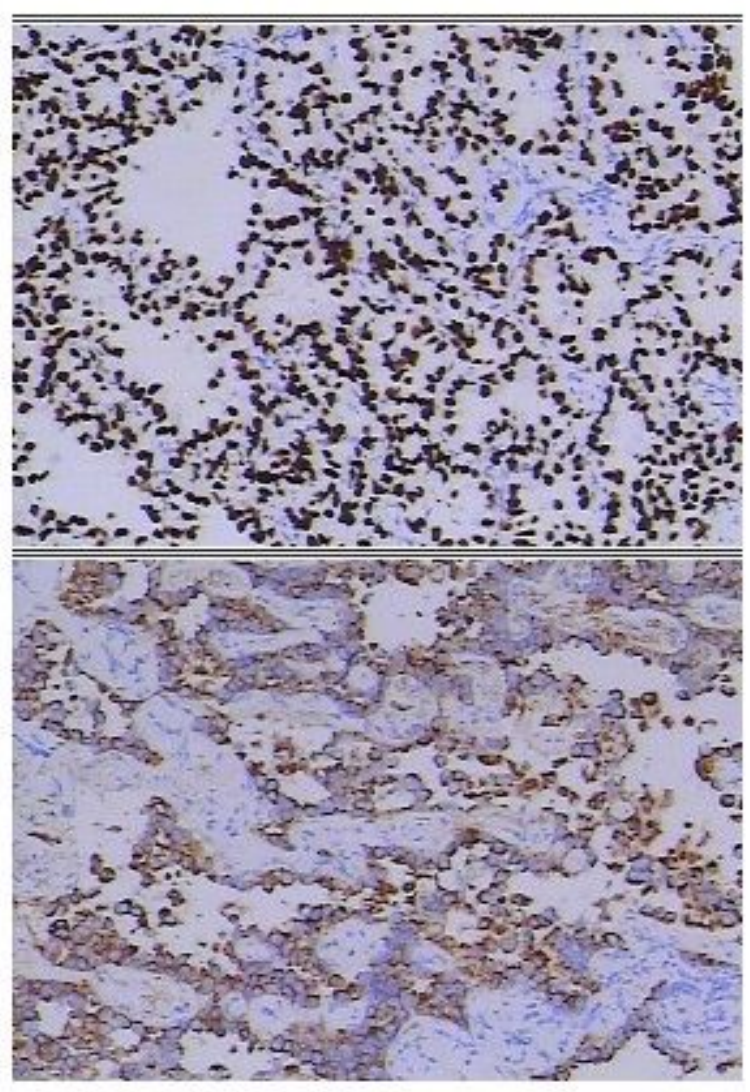

Figure 6

Immunohistochemistry of lung tumor 